\title{
Allele-specific DNA methylation analyses associated with siRNAs in Arabidopsis hybrids
}

\author{
CHEN ShaoXia $^{1}$, HE Hang $^{1 *} \&$ DENG XingWang ${ }^{1,2 *}$ \\ ${ }^{1}$ State Key Laboratory of Protein and Plant Gene Research, School of Life Sciences, Peking University, Beijing 100871, China; \\ ${ }^{2}$ Department of Molecular, Cellular and Developmental Biology, Yale University, New Haven, CT 06520-8104, USA
}

Received September 3, 2013; accepted September 18, 2013; published online April 2, 2014

\begin{abstract}
Accumulating evidence has suggested that epigenetic marks including DNA methylation, small RNA and histone modification may involve hybrid vigor in plants. However, knowledge about how epigenetic marks in hybrids regulate gene expression is still limited. Based on genome-wide DNA methylation landscapes of Arabidopsis thaliana Ler and C24 ecotypes and their reciprocal F1 hybrids which were obtained in our previous work, we analyzed allele-specific DNA methylation and distinguished cis- and trans-regulated DNA methylation in hybrids. Our study indicated that both cis- and trans-regulated DNA methylation played roles in hybrids, when cis-regulation played a major role in CG methylation and trans-regulation played major roles in CHG and CHH methylation. In addition, we observed correlations between trans-regulated DNA methylation and siRNA densities. Enriched siRNA regions were significantly concurrent with highly trans-regulated DNA methylation regions. Our results illustrated DNA methylation regulation patterns integrated with siRNAs in Arabidopsis hybrids, and shed light on understanding the mechanism of epigenetic reprogramming for hybrid vigor.
\end{abstract}

SNP, DNA methylation, siRNA, cis-, trans-, allele

Citation: $\quad$ Chen SX, He H, Deng XW. Allele-specific DNA methylation analyses associated with siRNAs in Arabidopsis hybrids. Sci China Life Sci, 2014, 57: 519-525, doi: 10.1007/s11427-014-4629-y

As an important epigenetic mark, DNA methylation plays many roles, including regulating gene expression, cell differentiation and system development process. DNA methylation changes in plant can affect the flowering, fertility, flowers and leaf morphology [1-8]. DNA methylation also follows some genetic rules. Morgan et al. [1] found that DNA methylation status of one locus in the Mus musculus inherited to the offspring, which determined the body color. Research in Arabidopsis also revealed that DNA methylation could be faithfully transmitted to the offspring $[2,3]$. And a large number of studies found DNA methylation was not constant during the heredity, but dynamically reprogramed [4-9]. The reprograming process of DNA methylation also varied among organisms: during Mus musculus

*Corresponding author (email: deng@pku.edu.cn; hehang@pku.edu.cn) embryonic development, through activation of parental DNA demethylation and repression of maternal DNA methylation, DNA methylation level decreased and then reprogramed which is different from two gametes [4-7]; the research on Danio rerio showed that the paternal DNA methylation patterns remained stable during early embryonic development, while the maternal DNA methylation changed dynamically [8,9]; in Arabidopsis thaliana, the maternal DNA methylation played a dominant role in early embryonic development, while the paternal DNA methylation contribution increased thereafter, which was associated with small RNAs [10]. Studies in mice indicated that cis-regulations affected most allele-specific DNA methylation in F1 [11]. Similar studies on allele-specific DNA methylation have also been carried out in Arabidopsis thaliana, Oryza sativa, Solanum lycopersicum and other spe- 
cies, when some of them indicated the associations between siRNA and allele-specific DNA methylation [12-18].

DNA methylation is known to build or maintain a helical or relaxed chromosomal state, while some 20-24-nt siRNAs can adjust the DNA methylation status of specific sites and regulate gene expression. RNA-directed DNA methylation $(\mathrm{RdDM})$ was firstly discovered in plant system [19]. Twenty-four nt siRNAs [20], which are generated by endoribonuclease DICER LIKE 3 (DCL3), are combined with ARGONAUTE 4 (AGO4) which contains PIWI domain and guide DOMAINS REARRANGED METHYLTRANSFERASE 2 (DRM2) activity [21-23] to catalyze de novo DNA methylation. Studies on rice indicated that allelespecific siRNA expression might mediate allele-specific DNA methylation [12]. Research on Arabidopsis and zebra fish confirmed the expression of 24-nt siRNAs is positively correlated with DNA methylation $[8,14]$.

We have used the next-generation sequencing method to achieve the whole-genome DNA methylation landscapes and small RNA expression in two Arabidopsis ecotypes and their reciprocal hybrids [17] in previous work. Here we analyzed allele-specific DNA methylation and siRNA expression in hybrids, illustrated the cis- and trans-regulation patterns of DNA methylation, and explored the roles of siRNAs in DNA methylation regulations.

\section{Materials and methods}

\subsection{Materials}

Experimental materials and sequencing data in our study were obtained from preliminary studies in our laboratory [17], when the original data were stored in the NCBI sequence database (accession number: GSE24658).

\subsection{Identification of single nucleotide polymorphisms (SNPs)}

Arabidopsis C24 and Ler genome sequences were downloaded from the 1001 Genomes Project. To discover SNPs between C24 and Ler genomes, C24 genome sequences were first cut into 38983 fragments of 3000 nucleotides length, and then mapped to Ler genome using Blastn [24], resulting in 38921 best match hits. After filtering 301 fragments that targeted to false chromosomes and 1117 fragments that targeted to Ler genomic repeat regions, we obtained 586902 SNPs from the remaining 37503 hits. Finally, we reconstructed $\mathrm{C} 24$ reference genome by replacing Ler genotype with C24 genotype in each SNP loci.

\subsection{Small RNA sequencing data processing}

Firstly, with original small RNA sequencing data, we processed quality controls, by trimming adapter sequences and low base quality sequences (base quality less than 5) in the
3 ' end, removing reads with more than $10 \%$ of " $N$ "s, removing reads with more than $50 \%$ of low quality bases, and only keeping sequences with longer than 16-nt length. Secondly, we aligned these clean reads to C24 and Ler genomes by bowtie [25], allowing no mismatches. The reads were separated according to parental genotypes in each SNP loci. In our study, the expression levels of small RNAs were represented by the numbers of small RNA reads in every 200-nt windows, moving through the genome by steps of 20-nt length.

\subsection{MethylC sequencing data processing}

Firstly, with original MethylC sequencing data, we processed quality controls in the same way as that in section 1.3. Secondly, we aligned the clean reads to C24 and Ler genomes using bismark [26]. The reads were separated according to parental genotypes in each SNP loci. In our study, DNA methylation intensities were calculated as dividing the number of methylated reads by the total read numbers on each $\mathrm{C} / \mathrm{G}$ sites. To detect differentially methylated sites, we filtered $\mathrm{C} / \mathrm{G}$ sites with less than four reads coverage and performed negatively binomial tests on two samples, with $P$-values less than 0.05 . To detect differentially methylated regions (DMRs), we selected regions with $P$-values less than 0.05 by Wilcox rank tests on two samples and with differences of methylation levels more than 0.1 between two samples in every 200-nt intervals, moving through the genome by steps of 20-nt length.

\section{Results and discussion}

\subsection{DNA methylation patterns and small RNA expres- sions on SNP loci}

There are abundant SNPs between genomes of the two Arabidopsis ecotypes C24 and Ler [27-29], which provided the feasibility for our studies. The C24 genome sequences were cut into 38983 fragments of 3000 nucleotides length, and then mapped to Ler genome using Blastn [24], resulting in 38921 best match hits. After filtering false hits, we obtained 586902 SNPs from the remaining 37503 hits, averagely one SNP per 200 bases. Of all SNPs, 53.1\% were "C/T" type (among "C/T", "G/A", "T/C" and "A/G" types), which was similar to that in rice [12].

Using next-generation sequencing, we obtained genome-wide DNA methylation and small RNA data with single-base resolution in Arabidopsis ecotype C24 and Ler and their reciprocal F1 hybrids (represented as FCL and FLC, Table S1 in Supporting Information). Among all reads mapped to both $\mathrm{C} 24$ and Ler genome reference, some overlapped those 586902 SNP loci: 7149819 for C24 (4.81\% of all C24 methylC reads), 8238223 for FCL (5.52\% of all FCL methylC reads), 7257946 for FLC (5.29\% of all FLC methylC reads) and 6931506 for Ler $(5.21 \%$ of all Ler 
methylC reads). After filtering $\mathrm{C} / \mathrm{G}$ sites with less than four reads coverage in each sample, 523278 sites remained, which comprised of 117928 CG-context, 108727 CHGcontext and $296623 \mathrm{CHH}$-context C/G sites. When applying the same strategy on small RNA sequencing data, the reads overlapped with SNP loci were: 613731 for C24 $3.00 \%$ of all C24 siRNA reads), 1825776 for FCL (7.63\% of all FCL siRNA reads), 1872542 for FLC (7.83\% of all FLC siRNA reads) and 785619 for Ler (3.80\% of all Ler siRNA reads). The mapping results indicated that we obtained abundant sequencing data for allele-specific DNA methylation and siRNA expression analysis.

MethylC sequencing data were aligned to both C24 to Ler genome using bowtie-based algorithm bismark [26]. $17.33 \%$ (7149819 of 1239415) of C24 methylC data were mapped to Ler genome sequence, and $6.60 \%$ (457407 of 6931506) of Ler methylC data were mapped to C24 genome sequence, indicating high accuracies of our strategy to detect allele-specific DNA methylation (Table S2 in Supporting Information). We also observed slight differences in genome-wide DNA methylation levels between Ler and C24 for CG sites, but equivalent levels for non-CG sites. The DNA methylation levels in hybrids of C24-origin and Ler-origin were similar to that of the original parents (Figure $1 \mathrm{~A})$.

\section{2 cis- and trans-regulation patterns of DNA methyla- tion in hybrids}

During the inheritance, offspring not only retain parental sequence information but also epigenetic information [2,30]. In this study, $\mathrm{C} / \mathrm{G}$ sites methylated in both parents were also methylated in hybrids mostly $(94.5 \%$ in FLC, and $94.8 \%$ in FCL). But for $\mathrm{C} / \mathrm{G}$ sites methylated in only one parent, only about half of them were methylated in hybrids: for sites methylated in Ler but unmethylated in C24, 49.47\% and $49.4 \%$ of them were detected methylated in FLC and FCL; for sites methylated in $\mathrm{C} 24$ but unmethylated in Ler, $37.26 \%$ and $38.09 \%$ of them were methylated in FLC and FCL (Table S3 in Supporting Information). And for $\mathrm{C} / \mathrm{G}$ sites methylated in neither parents, only $3.64 \%$ in FLC and $3.62 \%$ in FCL were methylated. These results indicated the consistent methylation patterns during the inheritance. Further comparisons were applied on methylation intensities on the same loci between the parents and the hybrids. As a result, from $2.77 \%$ to $3.18 \%$ of all $\mathrm{C} / \mathrm{G}$ sites were found significantly differently methylated between the parents and the hybrids. For the three different contexts of $\mathrm{C} / \mathrm{G}$ sites, the proportions were from $2.19 \%$ to $2.48 \%$ for CG-context, from $3.49 \%$ to $4.00 \%$ for CHG-context and from $2.68 \%$ to $3.16 \%$ for $\mathrm{CHH}-$ context methylation (only calculated on $\mathrm{C} / \mathrm{G}$ sites with more than four reads coverage, Figure 1B; Figure S1 in Supporting Information).

DNA methylation levels were known to regulate plant growth and development by dynamic changes. A series of research revealed that a large number of $\mathrm{C} / \mathrm{G}$ sites were differentially methylated in hybrids [12,31,32]. We found 28727, 24181 and 50555 differentially methylated sites in $\mathrm{CG}, \mathrm{CHG}$ and $\mathrm{CHH}$, respectively, between hybrids and parents. Among these sites, some of them were cis- regulated, which referred to the differential methylation caused by the genomic sequence variations, while others were trans-regulated, which referred to the differential methylation caused by upstream elements other than genomic sequences variations. We were able to effectively distinguish cis- from trans-regulation effects by comparing the allele-specific DNA methylation intensities between reciprocal hybrids and also between parents on the same sites (Figure 1C). When defining trans-regulated loci as sites with $P$-values $<0.05$ by negatively binomial tests on methylation differences between reciprocal hybrids and methylation differences between two parents, and defining cisregulated loci as other sites, we found $60.19 \%$ and $59.59 \%$ of the DNA differentially methylated CG sites were cisregulated in FLC and FCL, when $39.81 \%$ and $40.41 \%$ of the sites were trans-regulated in FLC and FCL, respectively. The proportions of trans-regulated sites were higher for CHG and $\mathrm{CHH}$ sites, accounting for $65.05 \%$ and $64.23 \%$ for CHG sites in FLC and FCL, respectively, and 75.66\% and $74.65 \%$ for $\mathrm{CHH}$ sites in FLC and FCL, respectively (Figure 1C; Table S4 in Supporting Information). These results implied the different regulated methylation modes on different $\mathrm{C} / \mathrm{G}$ contexts. Examples for cis- and transregulated sites are illustrated in Figure S2 in Supporting Information.

\section{3 siRNAs might play roles in DNA methylation reg- ulations in hybrids}

Differential methylated regions (DMRs) were suggested to regulate gene expression [12]. Using moving windows of 200-nt length throughout the genome by 20-nt step, we applied Wilcox rank tests within these windows to detect the DMRs between two parents and also between two alleles in hybrids. Our strategy resulted in 55759 DMRs (28374 for C24<Ler, and 27385 for C24>Ler) between two parents, 39338 DMRs (26244 for C24 allele<Ler allele, and 13094 for C24 allele>Ler allele) between two alleles in FLC, and 40561 DMRs (28459 for C24 allele<Ler allele, and 12102 for $\mathrm{C} 24$ allele>Ler allele) between two alleles in FCL. Then three groups of regions were selected for further studies: (i) not only DMRs between two parents, but also DMRs between two alleles in hybrids. Additionally, the DMRs between parents and between two alleles in hybrids were consistent (C24>Ler for two comparisons, or C24<Ler for two comparisons); (ii) DMRs between two parents, but not DMRs between two alleles in hybrids; (iii) not DMRs between two parents, but DMRs between two alleles in hybrids. Among these regions, only those belonging to the first group were cis-regulated, and the others were trans- 

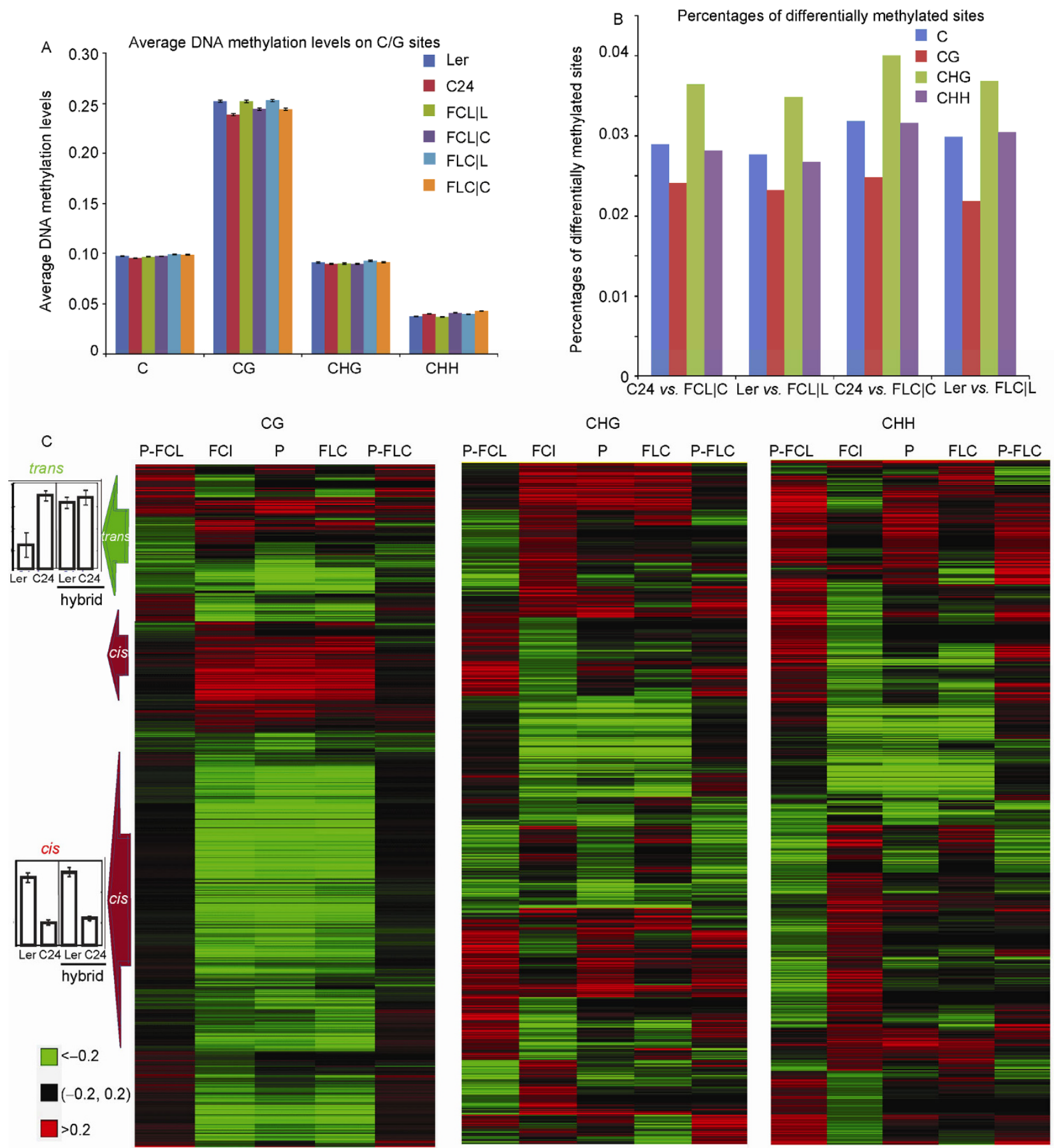

Figure 1 Regulation patterns of DNA methylation in Ler, C24 and reciprocal hybrids. A, Average DNA methylation levels on C/G sites and different contexts for parents and hybrids. B, Percentages of differentially methylated sites in four comparisons: between C24 and FCL when mapping to C24 genome, between Ler and FCL when mapping to Ler genome, between C24 and FLC when mapping to C24 genome, and between Ler and FLC when mapping to Ler genome. C, Heatmaps of DNA differentially methylated sites. P columns represent the differences between parents C24 and Ler, FCL and FLC columns represent allelic differences of DNA methylation in FCL and FLC, respectively, P-FCL columns represent differences between P and FCL values, and P-FLC columns represent differences between P and FLC values.

regulated. The numbers of regions belonging to three groups were 9452, 37499 and 21078 in FCL, and 10171, 36635 and 21473 in FLC (Table S5 in Supporting Information).

siRNAs were suggested as trans-acting elements for
DNA methylation, which were mediated by RdDM pathway to guide DNA methylation modification [19]. In this study, we selected DMRs belonging to above three groups with an additional condition: DNA methylation intensities for Ler allele were higher than that for C24 allele. Then the DMRs 
in each group were further divided into five sets, by counting the proportions of sites with methylation intensities for Ler allele more than that for $\mathrm{C} 24$ allele within every regions, from $50 \%-60 \%$ to $90 \%-100 \%$ (Figure $2 \mathrm{~A}$ ). We found that in group (i), siRNA densities were stable among those five sets, but higher than that of the genome-wide average level. But in group (ii) and (iii), with enhanced methylation differences between Ler and C24 alleles, siRNA densities increased significantly. These indicated that cis-regulated DMRs required accumulated siRNAs, and trans-regulated DMRs were positively correlated with siRNA densities (Figure 2A). Furthermore, in Figure 2B, proportions of DMRs with more than $75 \%$ siRNAs coming from Ler were calculated. Positive correlations were discovered between allele-specific siRNA expression and allele-specific DNA methylation for cis-regulated DMRs, but not for transregulated DMRs. These implied that siRNAs and DNA methylation were both cis-regulated in group (i) regions, but siRNAs played trans-acting roles in DNA methylation changes in group (ii) and (iii) regions. When comparing group (i) regions with group (iii) regions, we suggested that in DMRs between parents, methylation patterns in hybrids were determined by siRNA densities: if siRNAs were differentially expressed, DNA methylation would be cisregulated in hybrids; otherwise, DNA methylation would be trans-regulated in hybrids (Figure 2C).
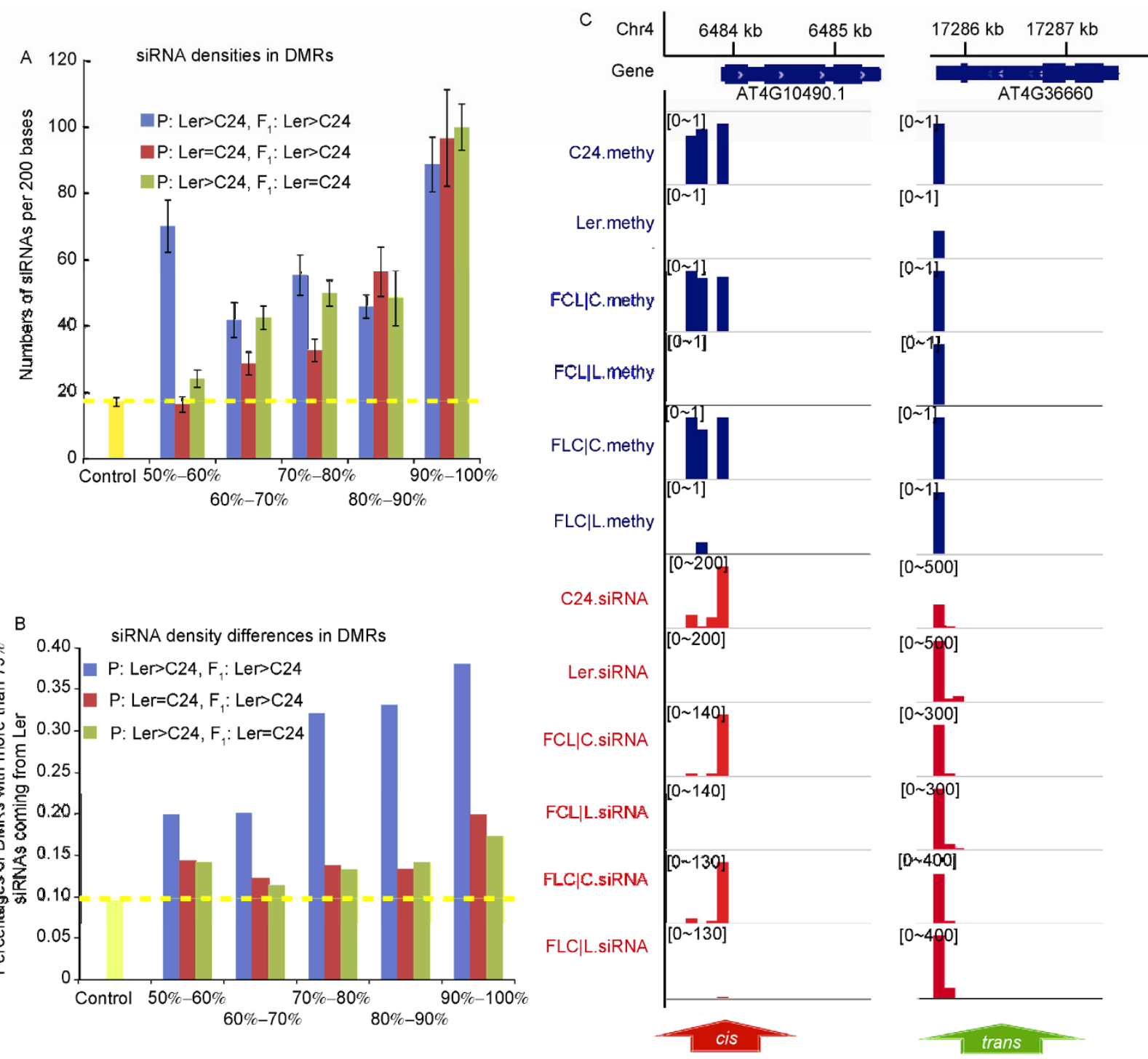

Figure 2 Correlations between differentially methylated regions (DMRs) and siRNAs. A, siRNA densities in DMRs. The $x$-axis represents the percentages of sites methylated higher in Ler than that in C24. The $y$-axis represents numbers of siRNAs per 200 bases. The control column represents genome regions other than DMRs. B, siRNA density differences in DMRs. The $x$-axis represents the percentages of sites methylated higher in Ler than that in C24. The $y$-axis represents percentages of DMRs with more than 75\% siRNAs coming from Ler. Blue and red lines represent percentage of DMRs with more than $75 \%$ siRNAs coming from Ler in hybrids. Green lines represent percentage of DMRs with more than $75 \%$ siRNAs coming from Ler in parents. The control column represents genome regions other than DMRs. C, Examples for cis-regulated and trans-regulated DMRs with siRNAs. 


\section{Conclusion}

In this study, we analyzed genome-wide allele-specific DNA methylation for Arabidopsis ecotypes C24 and Ler and their reciprocal hybrids. Firstly, we found that genome-wide methylation levels were about $25 \%, 10 \%$ and $5 \%$ for $\mathrm{CG}, \mathrm{CHG}$ and $\mathrm{CHH}$, respectively (Figure $1 \mathrm{~A}$ ), which were similar in both parents and hybrids. We mapped DNA methylation data of four samples to both C24 and Ler genome references. The results were high for mappings to self-origin genomes (80.5\% when mapping C24 data to C24 genome, and $96.12 \%$ when mapping Ler data to Ler genome), indicating that we had abundant sequencing data and applied reliable strategy for allele-specific DNA methylation analysis.

We found that most $\mathrm{C} / \mathrm{G}$ sites in hybrids inherited parental methylation patterns (from $96.00 \%$ to $97.81 \%$, Figure 1B). For differentially methylated sites between parents or hybrids, we distinguished them into cis-regulated and trans-regulated sites by comparing the allelic DNA methylation differences between two parents with that between reciprocal hybrids. Thus, we observed the major role of cis-regulation for CG sites $(60.19 \%)$ but minor roles for non-CG sites (24.34\% for CHG and $25.35 \%$ for CHH sites, Figure 1C; Table S4 in Supporting Information).

Associations were further revealed between siRNA densities and DNA methylation changes in DMRs. cisregulated siRNAs were found concurrent with cis-regulated DMRs, while siRNA densities were found positively correlated with trans-regulated DMRs. More interestingly, methylation patterns in hybrids seemed to be determined by siRNAs: cis-regulated siRNAs in parents resulted in cisregulated DNA methylation in hybrids, and trans-regulated siRNAs in parents resulted in trans-regulated DNA methylation in hybrids (Figure 2), implying that siRNAs played key roles in DNA methylation reprogramming in hybrids with RdDM pathway.

This work was supported by Ministry of Science and Technology of China (2013CBA01402), Ministry of Science and Technology of China (2012CB910900), Ministry of Agriculture of China (2010ZX08010-003), and Peking University 985 Program.

1 Morgan HD, Sutherland HG, Martin DI, Whitelaw E. Epigenetic inheritance at the agouti locus in the mouse. Nat Genet, 1999, 23: 314-318

2 Schmitz RJ, Schultz MD, Lewsey MG, O’Malley RC, Urich MA, Libiger O, Schork NJ, Ecker JR. Transgenerational epigenetic instability is a source of novel methylation variants. Science, 2011, 334: 369-373

3 Becker C, Hagmann J, Müller J, Koenig D, Stegle O, Borgwardt K, Weigel D. Spontaneous epigenetic variation in the Arabidopsis thaliana methylome. Nature, 2011, 480: 245-249

4 Wossidlo M, Nakamura T, Lepikhov K, Marques CJ, Zakhartchenko V, Boiani M, Arand J, Nakano T, Reik W, Walter J. 5-Hydroxymethylcytosine in the mammalian zygote is linked with epigenetic reprogramming. Nat Commun, 2011, 2: 241
5 Gu TP, Guo F, Yang H, Wu HP, Xu GF, Liu W, Xie ZG, Shi L, He X, Jin SG, Iqbal K, Shi YG, Deng Z, Szabó PE, Pfeifer GP, Li J, Xu GL. The role of Tet3 DNA dioxygenase in epigenetic reprogramming by oocytes. Nature, 2011, 477: 606-610

6 Inoue A, Zhang Y. Replication-dependent loss of 5-hydroxymethylcytosine in mouse preimplantation embryos. Science, 2011, 334: 194

7 Smith ZD, Chan MM, Mikkelsen TS, Gu H, Gnirke A, Regev A, Meissner A. A unique regulatory phase of DNA methylation in the early mammalian embryo. Nature, 2012, 484: 339-344

8 Jiang L, Zhang J, Wang JJ, Wang L, Zhang L, Li G, Yang X, Ma X, Sun X, Cai J, Zhang J, Huang X, Yu M, Wang X, Liu F, Wu CI, He C, Zhang B, Ci W, Liu J. Sperm, but not oocyte, DNA methylome is inherited by zebrafish early embryos. Cell, 2013, 153: 773-784

9 Potok ME, Nix DA, Parnell TJ, Cairns BR. Reprogramming the maternal zebrafish genome after fertilization to match the paternal methylation pattern. Cell, 2013, 153: 759-772

10 Autran D, Baroux C, Raissig MT, Lenormand T, Wittig M, Grob S, Steimer A, Barann M, Klostermeier UC, Leblanc O, Vielle-Calzada JP, Rosenstiel P, Grimanelli D, Grossniklaus U. Maternal epigenetic pathways control parental contributions to Arabidopsis early embryogenesis. Cell, 2011, 145: 707-719

11 Blewitt ME, Gendrel AV, Pang Z, Sparrow DB, Whitelaw N, Craig JM, Apedaile A, Hilton DJ, Dunwoodie SL, Brockdorff N, Kay GF, Whitelaw E. SmcHD1, containing a structural-maintenance-ofchromosomes hinge domain, has a critical role in $\mathrm{X}$ inactivation. Nat Genet, 2008, 40: 663-669

12 Chodavarapu RK, Feng S, Ding B, Simon SA, Lopez D, Jia Y, Wang GL, Meyers BC, Jacobsen SE, Pellegrini M. Transcriptome and methylome interactions in rice hybrids. Proc Natl Acad Sci USA, 2012, 109: 12040-12045

13 Greaves IK, Groszmann M, Ying H, Taylor JM, Peacock WJ, Dennis ES. Trans chromosomal methylation in Arabidopsis hybrids. Proc Natl Acad Sci USA, 2012, 109: 3570-3575

14 Groszmann M, Greaves IK, Albertyn ZI, Scofield GN, Peacock WJ, Dennis ES. Changes in 24-nt sRNA levels in Arabidopsis hybrids suggest an epigenetic contribution to hybrid vigor. Proc Natl Acad Sci USA, 2011, 108: 2617-2622

15 Greaves I, Groszmann M, Dennis ES, Peacock WJ. Trans-chromosomal methylation. Epigenetics, 2012, 7: 800-805

16 He G, Zhu X, Elling AA, Chen L, Wang X, Guo L, Liang M, He H, Zhang H, Chen F, Qi Y, Chen R, Deng XW. Global epigenetic and transcriptional trends among two rice subspecies and their reciprocal hybrids. Plant Cell, 2010, 22: 17-33

17 Shen H, He H, Li J, Chen W, Wang X, Guo L, Peng Z, He G, Zhong S, Qi Y, Terzaghi W, Deng XW. Genome-wide analysis of DNA methylation and gene expression changes in two Arabidopsis ecotypes and their reciprocal hybrids. Plant Cell, 2012, 24: 875-892

18 Shivaprasad PV, Dunn RM, Santos BA, Bassett A, Baulcombe DC Extraordinary transgressive phenotypes of hybrid tomato are influenced by epigenetics and small silencing RNAs. EMBO J, 2012, 31: 257-266

19 Wassenegger M, Heimes S, Riedel L, Sänger HL. RNA-directed de novo methylation of genomic sequences in plants. Cell, 1994, 76: 567-576

20 Henderson IR, Zhang X, Lu C, Johnson L, Meyers BC, Green PJ, Jacobsen SE. Dissecting Arabidopsis thaliana DICER function in small RNA processing, gene silencing and DNA methylation patterning. Nat Genet, 2006, 38: 721-725

21 Henderson IR, Jacobsen SE. Epigenetic inheritance in plants. Nature, 2007, 447: 418-824

22 Law JA, Jacobsen SE. Molecular biology. Dynamic DNA methylation. Science, 2009, 323: 1568-1569

23 Law JA, Jacobsen SE. Establishing, maintaining and modifying DNA methylation patterns in plants and animals. Nat Rev Genet, 2010, 11: 204-220

24 Camacho C, Coulouris G, Avagyan V, Ma N, Papadopoulos J, Bealer $\mathrm{K}$, Madden TL. BLAST+: architecture and applications. BMC Bioinformatics, 2009, 10: 421

25 Langmead B, Trapnell C, Pop M, Salzberg SL. Ultrafast and 
memory-efficient alignment of short DNA sequences to the human genome. Genome Biol, 2009, 10: R25

26 Krueger F, Andrews SR. Bismark: a flexible aligner and methylation caller for Bisulfite-Seq applications. Bioinformatics, 2011, 27: 1571-1572

27 Schneeberger K, Ossowski S, Ott F, Klein JD, Wang X, Lanz C, Smith LM, Cao J, Fitz J, Warthmann N, Henz SR, Huson DH, Weigel D. Reference-guided assembly of four diverse Arabidopsis thaliana genomes. Proc Natl Acad Sci USA, 2011, 108: 10249-10254

28 Gan X, Stegle O, Behr J, Steffen JG, Drewe P, Hildebrand KL, Lyngsoe R, Schultheiss SJ, Osborne EJ, Sreedharan VT, Kahles A, Bohnert R, Jean G, Derwent P, Kersey P, Belfield EJ, Harberd NP, Kemen E, Toomajian C, Kover PX, Clark RM, Rätsch G, Mott R. Multiple reference genomes and transcriptomes for Arabidopsis thaliana. Nature, 2011, 477: 419-423
29 Cao J, Schneeberger K, Ossowski S, Günther T, Bender S, Fitz J, Koenig D, Lanz C, Stegle O, Lippert C, Wang X, Ott F, Müller J, Alonso-Blanco C, Borgwardt K, Schmid KJ, Weigel D. Wholegenome sequencing of multiple Arabidopsis thaliana populations. Nat Genet, 2011, 43: 956-963

30 Stouder C, Paoloni-Giacobino A. Transgenerational effects of the endocrine disruptor vinclozolin on the methylation pattern of imprinted genes in the mouse sperm. Reproduction, 2010, 139: 373-379

31 Schilling E, El Chartouni C, Rehli M. Allele-specific DNA methylation in mouse strains is mainly determined by cis-acting sequences. Genome Res, 2009, 19: 2028-2035

32 Xie W, Barr CL, Kim A, Yue F, Lee AY, Eubanks J, Dempster EL, Ren B. Base-resolution analyses of sequence and parent-of-origin dependent DNA methylation in the mouse genome. Cell, 2012, 148: 816-831

Open Access This article is distributed under the terms of the Creative Commons Attribution License which permits any use, distribution, and reproduction in any medium, provided the original author(s) and source are credited.

\section{Supporting Information}

Table S1 Numbers of raw reads for small RNA-seq and methylC-seq

Table S2 Numbers of reads perfectly aligned to C24 genome and Ler genome for small RNA-seq and methylC-seq

Table S3 Methylated regions in hybrids but unmethylated in parents

Table S4 Differentially methylated sites in reciprocal hybrids by cis- and trans-regulations

Table S5 DMRs in reciprocal hybrids by cis- and trans-regulations

Figure S1 Percentages of differentially methylated sites by $c i s$ - and trans-regulations.

Figure S2 Examples for cis- and trans-DMRs.

The supporting information is available online at life.scichina.com and link.springer.com. The supporting materials are published as submitted, without typesetting or editing. The responsibility for scientific accuracy and content remains entirely with the authors. 